\title{
The comparative effectiveness of demineralized bone matrix, beta-tricalcium phosphate, and bovine-derived anorganic bone matrix on inflammation and bone formation using a paired calvarial defect model in rats
}

This article was published in the following Dove Press journal:

Clinical, Cosmetic and Investigational Dentistry

30 September 201I

Number of times this article has been viewed

\author{
Ahad Khoshzaban 1,2,3 \\ Shahram Mehrzad' \\ Vida Tavakoli ${ }^{2}$ \\ Saeed Heidari Keshel ${ }^{2}$ \\ Gholam Reza Behrouzi² \\ Maryam Bashtar ${ }^{2}$ \\ 'Iranian Tissue Bank Research and \\ Preparation Center, Imam Khomeini \\ Hospital Complex, ${ }^{2}$ Stem Cells \\ Preparation Unit, Eye Research \\ Center, Farabi Hospital, Tehran \\ University of Medical Science, \\ ${ }^{3}$ Dental Bio Material Department, \\ Tehran University of Medical Science, \\ Faculty of Dentistry, Tehran, Iran
}

Background: In this study, the effectiveness of Iranian Tissue Bank-produced demineralized bone matrix (ITB-DBM), beta-tricalcium phosphate ( $\beta$ TCP), and Bio-Oss ${ }^{\circledR}$ (Geistlich Pharma AG, Wolhusen, Switzerland) were evaluated and compared with double controls. The main goal was to measure the amount of new bone formation in the center of defects created in rat calvaria. Another goal was to compare the controls and evaluate the effects of each treatment material on their adjacent untreated (control) defects.

Methods: In this study, 40 male Wistar rats were selected and divided into four groups, In each group, there were ten rats with two defects in their calvarias; one of them is considered as control and the other one was treated with ITB-DBM (group 1), BIO-OSS (group2), and BTCP (group 3), respectively. But in group 4, both defects were considered as control. The amount of inflammation and new bone formation were evaluated at 4 and 10 weeks. In the first group, one defect was filled with ITB-DBM; in the second group, one defect was filled with Bio-Oss; in the third group, one defect was filled with $\beta$ TCP; and in the fourth group, both defects were left unfilled. Zeiss microscope (Carl Zeiss AG, Oberkochen, Germany) and Image Tool $^{\circledR}$ (version 3.0; University of Texas Health Science Center at San Antonio, San Antonio, TX) software were used for evaluation. SPSS Statistics (IBM Corp, Somers, NY) was used for statistical analysis.

Results: Maximum bone formation at 4 and 10 weeks were observed in the ITB-DBM group $(46.960 \% \pm 4.366 \%, 94.970 \% \pm 0.323 \%)$, which had significant difference compared with the other groups $(P<0.001)$. Ranking second was the Bio-Oss group and third, the $\beta$ TCP group. Bone formation in the group with two unfilled defects was much more significant than in the other controls beside the Bio-Oss and $\beta$ TCP after 10 weeks $(29.1 \pm 2.065,29.05 \pm 1.649)$, while this group had the least bone formation compared with the other controls at week 4 $(2.100 \% \pm 0.758 \%, 1.630 \% \pm 0.668 \%, P<0.001)$.

Conclusion: Overall, the ITB-DBM group showed the best results, although the results for other experimental groups were unfavorable. The authors conclude that human DBM (ITBDBM) should be offered as an alternative for bone regeneration in animals, such as horses, as well as in humans, especially for jaw reconstruction. In relation to bone regeneration in control defects, the effect of experimental material on controls was apparent during the initial weeks.

Keywords: $\beta$ TCP, Bio-Oss, bone regeneration, DBM 


\section{Introduction}

Regeneration of bone defects is one of the most important aims of researchers in the field of regenerative medicine. Tumors, bone cysts, osteomyelitis, and osteolysis, for example, can produce large bone defects, so different methods are being investigated to reconstruct these defects.

Autogenous cancellous bone graft is the gold standard and the most effective bone graft material, possessing the necessary characteristics for natural bone are osteointegration, osteoconduction, osteoinduction, and osteogenesis. An autogenous cancellous bone graft is usually obtained from the iliac crest and is the mainstay of osteogenic material. There is no risk of host rejection or disease transmission. ${ }^{1,2}$ Although autologous bone grafts are routinely used to heal large bone defects, the disadvantages of this intervention include extensive surgery, limited graft quantity, donor site pain, and morbidity, which have led to the development of improved methods of bone regeneration. ${ }^{3,4}$

An alternative, Allograft, has the same characteristics as autograft but is acellular. The allograft possesses osteoinductive properties only in the demineralized form. ${ }^{5}$ The complications associated with allograft are fracture, nonunion, bacterial infection, and viral transmission (however, viral transmission has been restricted with new molecular methods). The advantages of allograft include its availability and the avoidance of morbidity associated with harvesting autogenous graft. Allografts are of particular importance when there are large bone defects that require structural support or when inadequate autogenous graft volume is available. ${ }^{5}$

Some synthetic bone graft substitutes that are currently used may have bioactive characteristics. Many animal and clinical tests have been conducted to evaluate allograft materials and demineralized bone matrix (DBM) has been found to be an effective bone graft substitute in a variety of osseous defects. In fact, DBM is a form of allogenic bone graft substitute material. DBM is composed of insoluble collagen and proteins that are noncollagenous in nature and have low concentrations of growth factors. ${ }^{6}$ The decalcification process that occurs in bone products contributes to the exposition of proteins (collagen and noncollagen). This feature gives rise to another activity in bone regeneration. Indeed, the osteoinduction feature of these materials can appear by omitting $90 \%$ of calcified materials. DBM has been widely used in different clinical applications in powder form, particles of various sizes, large segments, gels, putties, and other composites. The primary osteoinductive component of DBM is a series of low-molecular-weight glycoproteins, which includes bone morphogenetic proteins (BMPs). ${ }^{7-9}$
The use of materials containing different types of growth factors (eg, BMP) is common. The history of BMPs began with the observation that DBM is able to induce ectopic bone formation in subcutaneous and intramuscular pockets in rodents. In several studies, histology revealed that the new bone was generated by osteoconductivity of DBM. ${ }^{8}$ Histological and biochemical analyses show that cartilage appears 5-10 days after implantation of active DBM. This cartilage mineralizes by days $7-14$ and is subsequently replaced with bone. ${ }^{10-12}$ After 21 days, hematopoietic bone marrow formation can be observed. These cellular events observed after DBM implantation mimic embryonic bone development and normal fracture repair. ${ }^{8}$

In addition to the inductive effect of the proteins in DBM, the collagen structure plays an osteoconductive role and supports new bone formation..$^{7-9}$ DBM has a number of additional advantages that make it an attractive bone graft alternative. It is cost-effective and readily available from the tissue banks. The demineralization process destroys the antigenic materials in bone, making DBM less immunogenic than mineralized allograft. ${ }^{9}$

At present, the available xenografts used clinically for defects are Bio-Oss ${ }^{\circledR}$ (Geistlich Pharma AG, Wolhusen, Switzerland), Osteograf/N, Interpore $200^{\circledR}$ (Interpor Orthopaedics, Inc, Irvine, CA), and Biocoral ${ }^{\circledR}$ (natural carbonate; Biocoral Inc, Saint Gonnéry, France).${ }^{13}$ Bio-Oss is bovine bone that has had organic substances extracted; the remaining structure with fine pores is the same as either natural bone, chemical compound or microstructure. ${ }^{14}$

A variety of artificial materials has been used over the centuries to fill bone defects. Synthetic bone grafts possess at most only two of the four characteristics of an ideal bone graft material (osteointegration and osteoconduction). Ideally, synthetic bone graft substitutes should be biocompatible, show minimal fibrotic reaction, undergo remodeling, and support new bone formation. ${ }^{5}$ From a mechanical point of view, synthetic bone graft substitutes should have a similar strength to that of the cortical/cancellous bone being replaced and a similar modulus elasticity to prevent fatigue fracture under cyclic loading. Synthetic materials that demonstrate some of these properties are composed of either calcium, silicon, or aluminum glasses: ${ }^{5}$ glass ionomers, aluminum oxide, calcium sulfate, calcium phosphate, beta-tricalcium phosphate ( $\beta \mathrm{TCP})$, synthetic hydroxyapatite, coralline hydroxyapatite, and calcium phosphate cements.

Although biomaterials have many advantages, they are not successfully absorbed in the body, cannot produce bony trabecules as natural bone, and are amorphous. Also, they are very weak at the site of connection between bone and implant. ${ }^{15}$ 
ßTCP was one of the earliest calcium phosphate compounds, which was used as a bone graft substitute that Albee and Morrison reported in 1920. ${ }^{2,5,16}$ BTCP is available in porous or solid form as either granules or blocks. Structurally similar to cancellous bone, porous $\beta$ TCP is brittle and weak under tension and shears but is resistant to compressive loads. Typically, it has been used in its granular porous form. $\beta T C P$ undergoes reabsorption via dissolution and fragmentation over a period of $6-18$ months. Unfortunately, there is always less bone volume produced than the volume of $\beta$ TCP reabsorbed. For this reason, the clinical use of $\beta \mathrm{TCP}$ has been adjunctive with other less-reabsorbable bone graft substitutes or as an expander for autogenous bone grafts. ${ }^{2,5,16}$

The search for the best graft materials for bone regeneration and bone graft substitutes continues. The best substances are those that affect cell activity and have similar biological and biomechanical characteristics to natural bone tissue. These materials are biocompatible, osteoconductive, and osteoinductive, and reinforce bone repair., ${ }^{5,17}$

The present study is performed to investigate the rate of bone induction of processed DBM in Iranian Tissue Bank Research and Preparation Center, Imam Khomeini Hospital, Tehran University of Medical Sciences. Bone powder is demineralized bone that has had all of its organic matter removed, and is available in different particle sizes. Bone powders at this center are produced in different sizes, from $75 \mu \mathrm{m}$ to the very large size (gross bone) used for various applications including dentistry, oral and maxillofacial (eg, face reconstruction after trauma), orthopedic (eg, severe bone reduction in sarcoma or another cancer), and neurosurgery due to bone regeneration.

In this animal study, bone remodeling and inflammation were evaluated in rat calvaria with a 6-mm defect (considered critical diameter). The effectiveness of Iranian Tissue Bank-produced DBM (ITB-DBM), $\beta$ TCP, and Bio-Oss were evaluated and compared. The effects of each treatment material on their adjacent untreated (control) defects were also evaluated.

\section{Methods}

The rat was used in this study as an animal model. Forty male rats (Wistar species, purchased from Pasteur Institute of Iran [Tehran, Iran], each weighing 250-300 g) were selected and divided into four groups, each consisting of ten rats. Following anesthesia, a vertical incision was made from the nose tip to between the ears (sagittal suture). Periosteum was removed and then two defects were made in the parietal bone with a trephine burr number 6 . One defect in all groups was left empty. In the first group, the other defect was filled with ITB-DBM. In the second group, the other defect was filled with Bio-Oss ${ }^{\circledR}$ (Geistlich, Wolhusen, Switzerland) bone powder; this material, according to the product description, is osteoconductive and remains in the body without degrading for 8-10 years. In the third group, the other defect was filled with $\beta$ TCP (ATLANTIC ${ }^{\circledR}$, Genta France; Medical Biomat, 69120 - Vaulx EN Velin). In the fourth group, both defects were left empty (to compare empty defects with and without consumer substances to obviate errors [in most studies, errors were observed due to the interference of added substances in control and test defects]).

Start time of the study was 1 week following the suture. After 4 weeks, three rats were sacrificed in each group, and 10 weeks later, the remaining seven rats were sacrificed. Initially, samples were fixed into formalin for 10 days for section preparation and then immersed in $10 \%$ formic acid for 20 days (tests were carried out every 3 days using a special needle to realize bone readiness for preparation of sections). The defects were then divided into two parts. Five sections were taken from each sample and stained with hematoxylin. Next, bone formation and inflammation were evaluated in the sections. Zeiss microscope (Carl Zeiss AG, Oberkochen, Germany) and Image Too ${ }^{\circledR}$ software were used for evaluation. Each field was examined with $10 \times, 20 \times$, and $40 \times$ magnification, and the amount of inflammation and bone formation was graded as follows: grade 1, very little or absent; grade 2, bone formation was more than 33\% of software ruler at the center of each field; grade 3 , bone formation was more than $50 \%$ of software ruler; grade 4 , bone formation contains the total length of software ruler. All slides were evaluated using Photoshop ${ }^{\circledR}$ software (Adobe Systems Inc, San Jose, CA). In current qualitative survey, all pixels in the image were obtained, and then the places of inflammation and bone formation were specified on the extracted pixels of the image. Finally, the percentage of pixels (the ratio of inflammation and bone regeneration to the whole sample) was obtained for each variant.

\section{Percentage calculation method for grading}

In this study, every slide was evaluated at $10 \times$ magnification. The defect center field was then adjusted at $40 \times$ magnification. The amount of bone formation in the center of the defect was measured. The number the grades described above for bone formation or inflammation was selected in each slide based on the ratio of the total pixels to the pixels, which represented inflammation and bone formation, and then agreed 
the number to enter in SPSS Statistics (IBM Corp, Somers, NY) tables. The numbers underwent nonparametric analysis, the tables were compared, and differences between each were obtained. For example, one slide had 1011.9 pixels and the pixels for each selected part were determined. Photoshop software showed that pathology sample (image) totally has a certain number of pixels. In this study, the ratio of the inflammation and bone regeneration to the whole image was intended to investigate. If 72.5 inflammation is $100 \%$, then 25 inflammation should be $34.4 \%$, expressed as $35 \%$.

The results are presented as mean and standard deviation. The data were analyzed using one-way analysis of variance (ANOVA) (Dunnett's T3 test). Comparison of data on the bone formation and inflammation was done using ANOVA. A $P$-value $\leq 0.05$ was considered significant.

\section{Results}

Overall, the ITB-DBM showed the best results, although the results for other experimental groups were unfavorable (Table 1). The inflammation at week 4 in the ITB-DBM group was less than the other groups $(41.530 \% \pm 3.702 \%$; $P<0.001)$. The Bio-Oss group had the least inflammation at week $4(58.400 \% \pm 2.847 \% ; P<0.001)$. The $\beta$ TCP group ranked fourth for the minimum inflammation at week 4 (74.900\% $\pm 32.975 \% ; P<0.001)$ after group 6 (control 3) $(74.83 \% \pm 3.13 \%)$. The difference between this group and control 3 was not significant $(P=1)$ (Figure 1).

Among the controls, control 3 had the minimum week-4 inflammation $(74.83 \% \pm 3.1 \% ; P<0.001)$; control 4

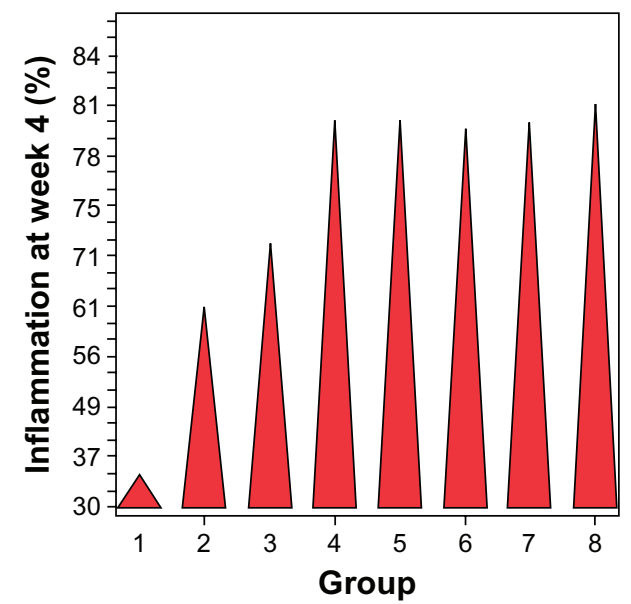

Figure I Inflammation at week 4 in the eight groups: group I, ITB-DBM-filled defect; group 2, Bio-Oss-filled defect; group 3, $\beta$ TCP-filled defect; group 4, control I (two empty defects); group 5, control 2 (two empty defects); group 6, control 3 (empty defect adjacent to ITB-DBM-filled defect); group 7, control 4 (empty defect adjacent to BioOss-filled defect); group 8, control 5 (empty defect adjacent to $\beta T C P$-filled defect). Abbreviations: $\beta T C P$, beta-tricalcium phosphate; ITB-DBM, Iranian Tissue Bankproduced demineralized bone matrix.

ranked second $(82.930 \% \pm 4.322 \%)$ and had no significant differences in comparison with all other controls except for control 3; control 1 ranked third $(83.530 \% \pm 3.014 \%)$; control 5 ranked fourth $(84.030 \% \pm 2.965 \%)$; and control 2 had the maximum week-4 inflammation $(85.760 \% \pm 3.626 \%)$, the differences between this group and the other controls were not significant except control $3(P<0.001)$. There were no significant differences with the other controls except control 3. Control 1 had no significant differences with the other controls except control $3(P<0.001)$ (Figure 1).

Table I Mean and SD of inflammation and bone formation at weeks 4 and 10

\begin{tabular}{|c|c|c|c|c|c|c|c|c|}
\hline & $\mathbf{I}^{\mathbf{a}}$ & $2^{b}$ & $3^{c}$ & $4^{d}$ & $5^{e}$ & $6^{f}$ & $7^{g}$ & $8^{\text {h }}$ \\
\hline \multicolumn{9}{|c|}{ Inflammation at week 4} \\
\hline Mean (\%) & 41.53 & 58.40 & 74.90 & 83.53 & 85.76 & 74.83 & 82.93 & 84.03 \\
\hline SD (\%) & 3.702 & 2.847 & 2.975 & 3.014 & 3.626 & 3.13 & 4.322 & 2.965 \\
\hline Min-Max (\%) & $32-48$ & $49-63$ & $70-81$ & $80-89$ & $80-91$ & 70-79 & $73-89$ & $80-89$ \\
\hline \multicolumn{9}{|c|}{ Inflammation at week 10} \\
\hline Mean (\%) & 13.6 & 19.58 & 25.24 & 28.91 & 28.85 & 22.27 & 23.41 & 25.78 \\
\hline SD (\%) & $\mathrm{I} .488$ & 0.955 & 2.533 & 1.7 & 1.721 & 2.425 & 1.619 & 2.056 \\
\hline Min-Max (\%) & $10-17$ & $18-22$ & $20-29$ & $24-34$ & $24-33$ & $19-30$ & $21-27$ & $23-31$ \\
\hline \multicolumn{9}{|c|}{ Bone formation at week 4} \\
\hline Mean (\%) & 46.96 & 25.26 & 12.83 & 2.10 & 1.63 & 7.63 & 4. 10 & 3.03 \\
\hline SD (\%) & 4.366 & 3.403 & 1.193 & 0.758 & 0.668 & 1.401 & I.184 & 0.85 \\
\hline Min-Max (\%) & $35-54$ & $20-31$ & $9-17$ & $1-4$ & $\mathrm{I}-3$ & $3-9$ & $2-6$ & $\mathrm{I}-5$ \\
\hline \multicolumn{9}{|c|}{ Bone formation at week 10} \\
\hline Mean (\%) & 94.97 & 81.80 & 47.72 & 29.10 & 29.05 & 24.58 & 24.61 & 26.47 \\
\hline SD (\%) & 3.323 & 3.998 & 2.728 & 2.065 & 1.649 & 5.467 & 3.298 & 2.435 \\
\hline Min-Max (\%) & $88-99$ & $75-89$ & $40-52$ & $24-35$ & $25-33$ & $19-39$ & $21-35$ & $21-34$ \\
\hline
\end{tabular}

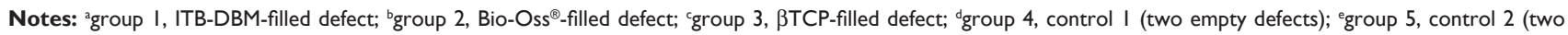
empty defects) fgroup 6, control 3 (empty defect adjacent to ITB-DBM-filled defect); ggroup 7, control 4 (empty defect adjacent to Bio-Oss-filled defect); ' $g r o u p ~ 8$, control 5 (empty defect adjacent to $\beta$ TCP-filled defect).

Abbreviations: $\beta$ TCP, beta-tricalcium phosphate; ITB-DBM, Iranian Tissue Bank-produced demineralized bone matrix; SD, standard deviation. 
With regards to bone formation at week 4 , the maximum mean was correlated with the ITB-DBM group $(46.960 \% \pm 4.366 \%)$ (Figure 2A). There were significant differences compared with the other groups, particularly Bio-Oss, $\beta$ TCP, and its control (control 3) $(P<0.001)$. The Bio-Oss group ranked second for the maximum bone formation $(25.260 \% \pm 3.403 \%)$. The differences between this group and others including $\beta \mathrm{TCP}$ and its control (control 4) were significant $(P<0.001)$. Ranking third for maximum bone formation was the $\beta$ TCP group $(12.830 \% \pm 1.931 \%)$ (Figure $2 B$ ). This group had significant differences with other groups, especially its control (control 5) $(P<0.001)$ (Figure 3).

Control 3 rats had the maximum week-4 bone formation compared with other controls $(7.630 \% \pm 1.401 \%$; $P<0.001)$. Ranking second in this category was control $4(4.100 \% \pm 1.184 \% ; P \leq 0.005)$. Control 5 ranked third $(3.03 \% \pm 0.85 \% ; P \leq 0.005)$. Among the controls,

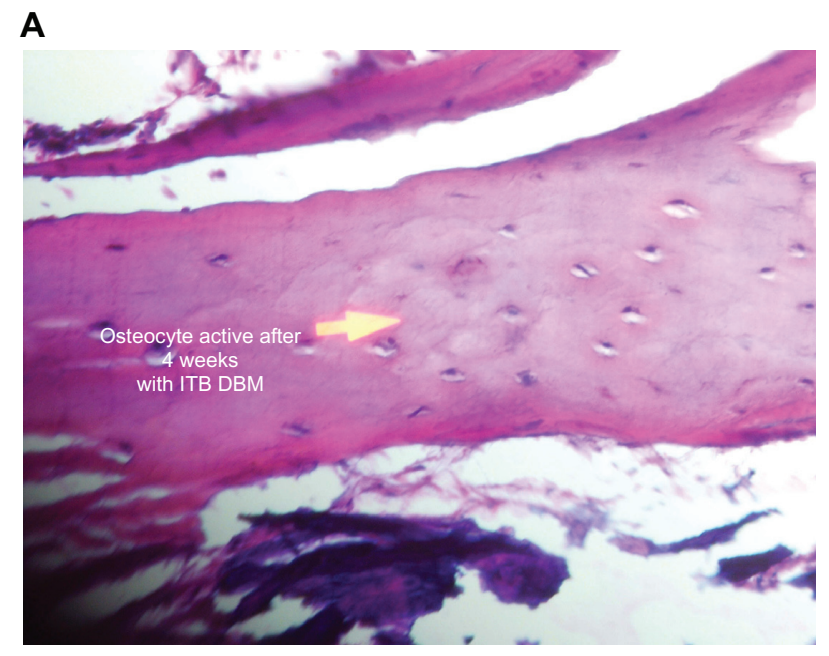

B

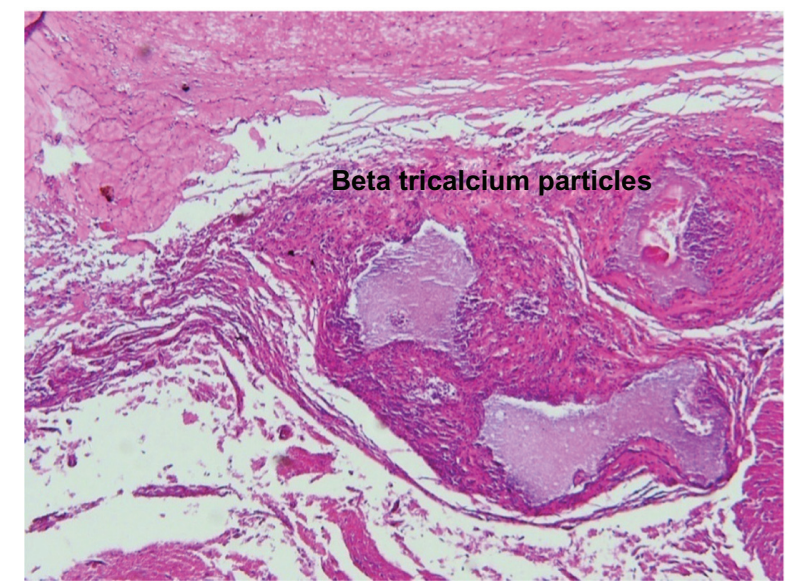

Figure 2 (A) Bone formation at week 4 in the ITB-DBM group. (B) Beta-tricalcium particles at the site of fibrosis tissue.

Abbreviation: ITB-DBM, Iranian Tissue Bank-produced demineralized bone matrix. the minimum bone formation was seen in controls 1 and 2 $(2.100 \% \pm 0.758 \%, 1.630 \% \pm 0.668 \%$, respectively). The difference between these two groups was not significant $(P=0.311)$ (Figure 3).

At week 10, the minimum inflammation was seen in group $1(13.600 \% \pm 1.488 \%)$. The differences between this group and other groups, including groups 2,3 , its control (group 6), and controls 1 and 2 were significant $(P<0.001)$. Ranking second for minimum week-10 inflammation was group $2(19.580 \% \pm 0.955 \%)$. It had a significant difference compared with other groups, especially group 3, its control (group 7), and controls 1 and $2(P<0.001)$. Group 3 ranked third $(25.240 \% \pm 2.533 \%)$ for the minimum week-10 inflammation, followed by groups $6(22.270 \% \pm 2.425 \%)$ and 7 $(23.410 \% \pm 1.619 \%)$. The significant differences were seen between group 3 and other groups $(P<0.001)$, except its control (group 8$)(P=0.991)$. But as mentioned, it had a significant difference with controls 1 and $2(P<0.001)$ (Figure 4).

Comparing the controls, control 3 had the minimum week-10 inflammation $(22.270 \% \pm 2.425 \%)$. Significant differences were seen with other controls including control 1,2 , and $5(P<0.001)$ and also control $4(P=0.037)$. Control 4 ranked second for the minimum week-10 inflammation $(23.410 \% \pm 1.619)$. There were significant differences between this group (control 4) and other controls, especially controls 1 and $2(P<0.001)$. Ranking third among the controls for the minimum week-10 inflammation was control $5(25.780 \% \pm 2.056 \%)$. Its differences with other controls, including control 1 and 2 , were significant $(P<0.001)$. Finally, the maximum week-10 inflammation was seen in the main controls (controls 1 and 2). The difference between these two groups was not significant $(P=1)$ (Figure 4).

The maximum week-10 bone formation was seen in the ITB-DBM group $(94.970 \% \pm 3.323 \%)$ (Figure $5 \mathrm{~A})$. The differences between this group and other groups, including $\beta T C P$, Bio-Oss, and control 3 were significant $(P<0.001)$. The Bio-Oss group ranked second for maximum week-10 bone formation $(81.800 \% \pm 3.998 \%$ ) (Figure 5B). There was a significant difference in comparison with others specially $\beta$-TCP and control $4(P<0.001)$. The $\beta$ TCP group ranked third $(47.720 \% \pm 2.728 \%)$ and had a significant difference with other groups, particularly control $5(P<0.001)$ (Figure 6).

Among the controls, at week 10, the maximum bone formation was in the main control groups with two empty defects; that is, control $1(29.100 \% \pm 2.065 \%)$ and control 2 $(29.050 \% \pm 1.649 \%)$. There was no significant difference between these two groups, but the differences between these two groups and the other controls were significant $(P<0.001)$. 


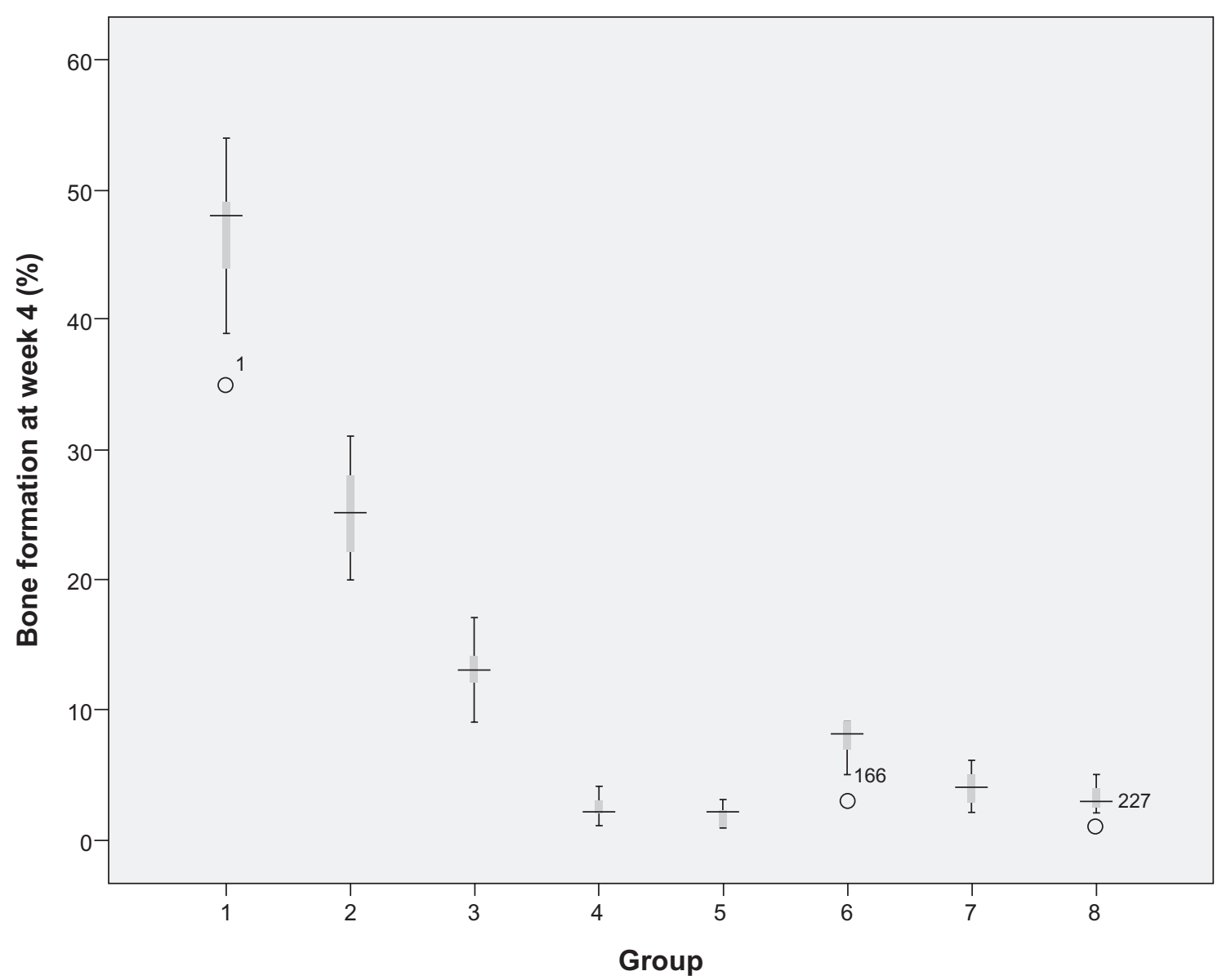

Figure 3 Bone formation at week 4 in the eight groups: group I, ITB-DBM-filled defect; group 2, Bio-Oss ${ }^{\circledR}$-filled defect; group 3, $\beta$ TCP-filled defect; group 4, control I (two empty defects); group 5, control 2 (two empty defects); group 6, control 3 (empty defect adjacent to ITB-DBM-filled defect); group 7, control 4 (empty defect adjacent to Bio-Oss-filled defect); group 8, control 5 (empty defect adjacent to $\beta$ TCP-filled defect).

Abbreviations: $\beta$ TCP, beta-tricalcium phosphate; ITB-DBM, Iranian Tissue Bank-produced demineralized bone matrix.

The next ranking positions for maximum week-10 bone formation were for controls 5,4 , and $3(26.470 \% \pm 2.435 \%$, $24.610 \% \pm 3.298 \%$, and $24.580 \% \pm 5.467 \%$, respectively). Control 3 had no significant differences compared with control 4 and control 5 ( $P=1, P=0.233$, respectively). Other differences between these controls and also controls 1 and 2 were significant (Figure 6).

\section{Discussion}

The main goal of this study was to measure the amount of new bone formation within the center of each bone defect. Defects of an appropriate size (6 $\mathrm{mm}$ diameter) were created, and the amount of inflammation and new bone formation was measured at weeks 4 and 10, in four main groups: group 1 (ITB-DBM), group 2 (Bio-Oss), group 3 ( $\beta$ TCP), and group 4 (two empty defects). Furthermore, beside each defect containing ITB-DBM, Bio-Oss, or $\beta$ TCP was an unfilled defect of the same size. These defects were the control defects. The inflammation and bone formation of the empty defects beside the defects containing bone stimulating materials (osteoconductive materials, Bio-Oss and $\beta$ TCP; or osteoinductive materials, ITB-DBM), were compared with two empty defects in the control group. This method (empty defect beside experimental defect) is often considered as the main control for comparison in experimental research.

An important question raised was whether the material of the experimental defect got transferred to its adjacent empty defect from circulation and animal movement, especially at the operation area, after periosteal approximation. In this study, the results show that the experimental defect materials significantly affected their adjacent empty defects, and the differences between these empty defects and those of group 4 were significant. In relation to bone regeneration in control defects, the effect of experimental defects on controls was apparent in the early weeks. Thus, inflammation and bone regeneration of the control defects were affected by their adjacent experimental defects. However, this effect gradually declined in later weeks because the bone formation of the control defects, unlike the experimental defects, was started from around the defects and not in the center. 


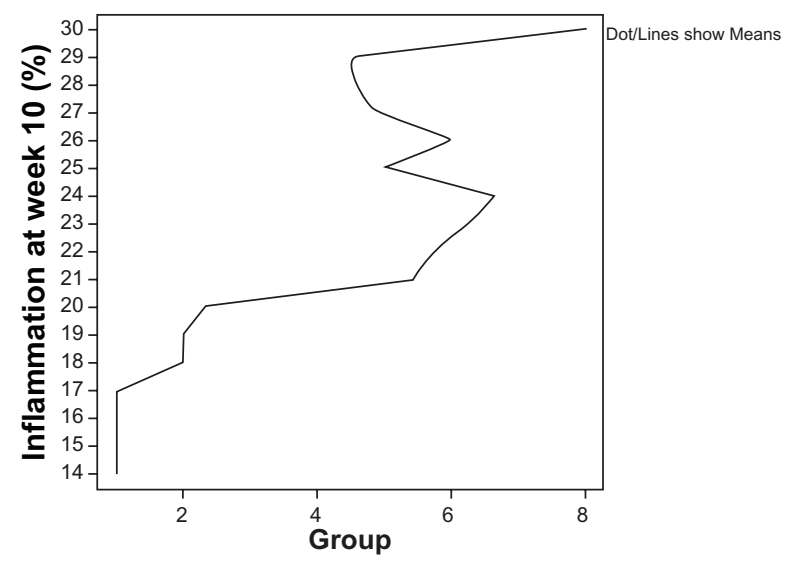

Figure 4 Inflammation at week 10 in the eight groups: group I, ITB-DBM-filled defect; group 2, Bio-Oss ${ }^{\circledR}$-filled defect; group 3, (TCP-filled defect; group 4, control I (two empty defects); group 5, control 2 (two empty defects); group 6, control 3 (empty defect adjacent to ITB-DBM-filled defect); group 7, control 4 (empty defect adjacent to Bio-Oss-filled defect); group 8, control 5 (empty defect adjacent to $\beta$ TCP-filled defect).

Abbreviations: $\beta$ TCP, beta-tricalcium phosphate; ITB-DBM, Iranian Tissue Bankproduced demineralized bone matrix.

After 10 weeks, the bone formation in the group with two empty defects was then significantly greater than for the other controls $(29.100 \% \pm 2.065 \%, 29.050 \% \pm 1.649 \%)$, while at week 4 , this group had the least bone formation compared with the other controls. These differences were statistically significant.

At week 4, the maximum bone formation occurred in the ITB-DBM group $(46.960 \% \pm 4.366 \%)$, which had significant difference in comparison with all groups $(P<0.001)$; while minimum bone formation occurred in group 4 (controls 1 and 2 in which both defects were empty) $(2.100 \% \pm 0.758 \%)$. The difference between the two empty defects was not significant $(P=0.311)$. At week 10 , the maximum bone formation occurred in the ITB-DBM group $(94.970 \% \pm 0.323 \%)$ and the differences in comparison with other groups were significant $(P<0.001)$.

The natural processes of bone repair in various places are different. They are dependent on complex situations and different materials, including osteoinductive or osteoconductive substances. The aim of bone regeneration is restoration of bone integrity. The bone of each zone on the basis of its efficiency and location requires specific regeneration. For example, cancellous bone, cortical bone, or mixed bone have different applications in different areas of the body. In several studies, especially in vivo tests, information about osteoinductive or osteoconductive effects of different materials have been histologically and mechanically surveyed. These results, along with support of other theories, were expanded in clinical practice. However, in some cases, clinical measurements are not consistent with histological findings. ${ }^{18}$ The use of various materials as a scaffold for acceleration in bone regeneration is a routine method in clinical practice. The main goal of the researchers by applying the bone substitutes is to activate the procedure of bone regeneration.

A multicenter study showed that after 6 months insertion of a $\beta$ TCP graft resulted in the formation of a stable bony bed suitable for the anchoring of dental implants. ${ }^{19}$

In 2007, Jassar et al evaluated the efficacy of various biomaterials in bone healing when they were used in bony surgical defects of the oral and maxillofacial region. ${ }^{20}$ Alloplastic grafts such as hydroxyapatite, $\beta$ TCP, and demineralized bovine bone were used. Resorbable porous grafts have shown good results in promoting bone healing in various surgical defects due to their inherent osteoconduction property; hence, they should be more commonly used. ${ }^{20}$

Many animal and clinical tests have been conducted to evaluate allograft materials, and DBM has been found to be an effective bone graft substitute in a variety of osseous defects. This material has been widely used in different clinical applications in the form of powder, particles of various sizes, large segments, gels, putties, and other composites. ${ }^{7-9}$ DBM has a number of additional advantages that make it an attractive bone graft alternative. It is cost-effective and readily available from tissue banks. The demineralization

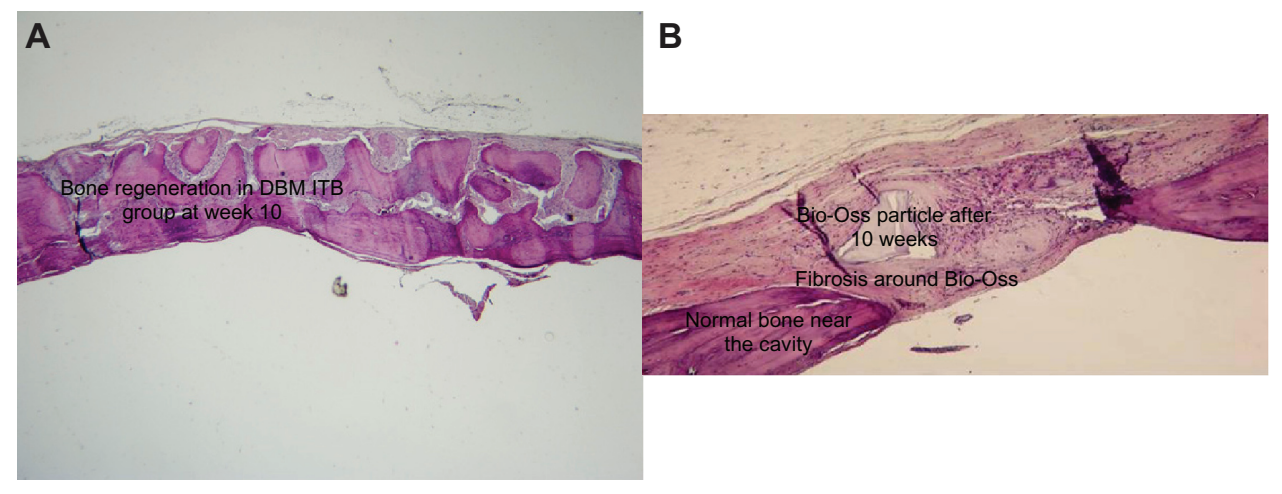

Figure 5 (A) Bone formation of ITB-DBM at week 10. (B) Bone formation of Bio-Oss at week 10. Abbreviation: ITB-DBM, Iranian Tissue Bank-produced demineralized bone matrix. 


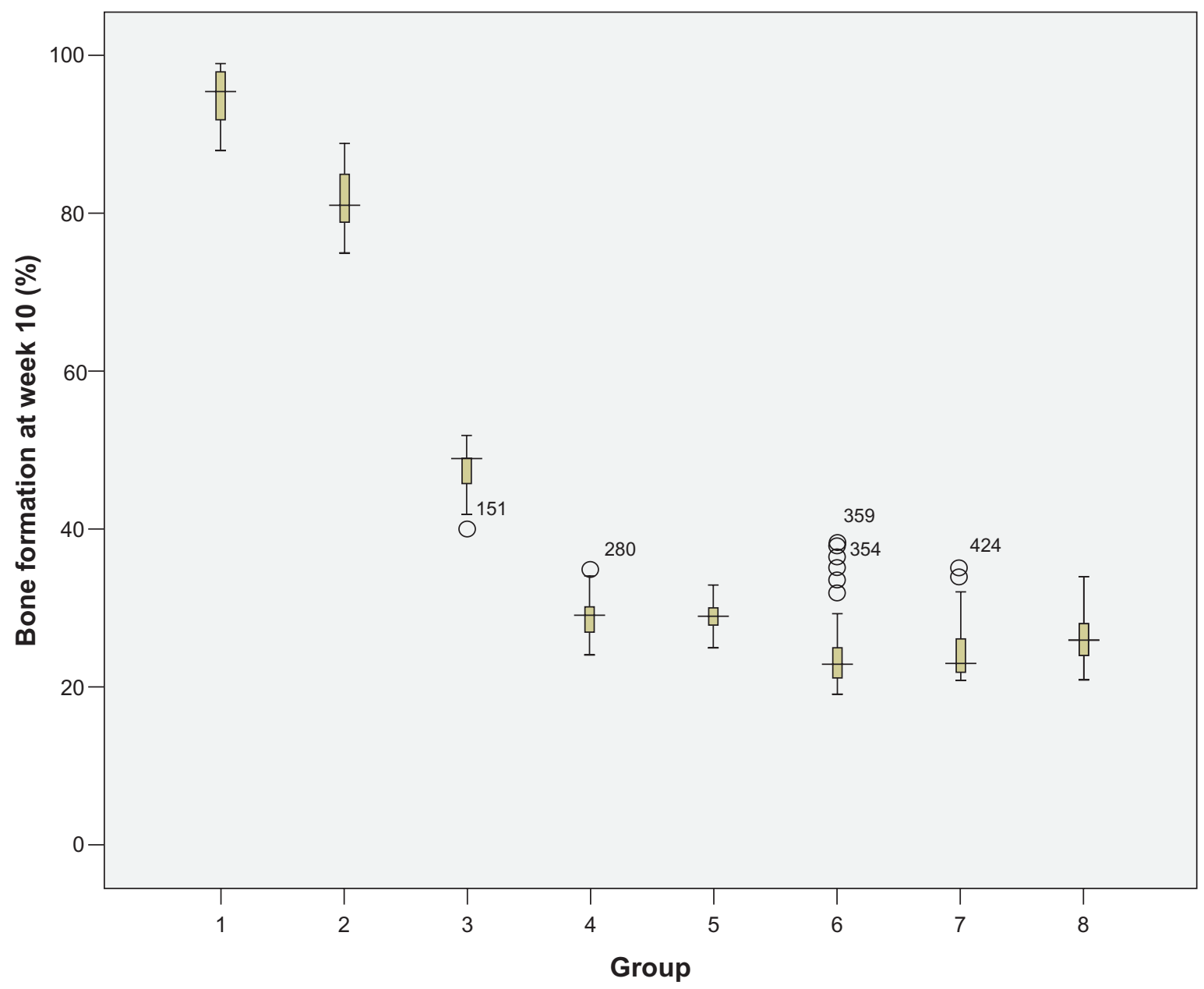

Figure 6 Bone formation at week 10 in the eight groups: group I, ITB-DBM-filled defect; group 2, Bio-Oss ${ }^{\circledR}$-filled defect; group 3 , $\beta$ TCP-filled defect; group 4, control I (two empty defects); group 5, control 2 (two empty defects); group 6, control 3 (empty defect adjacent to ITB-DBM-filled defect); group 7, control 4 (empty defect adjacent to Bio-Oss-filled defect); group 8, control 5 (empty defect adjacent to $\beta$ TCP-filled defect).

Abbreviations: $\beta T C P$, beta-tricalcium phosphate; ITB-DBM, Iranian Tissue Bank-produced demineralized bone matrix.

process destroys the antigenic materials in bone, making DBM less immunogenic than mineralized allograft. ${ }^{9}$ Han et al confirm that removing lipids from DBM during the process of preparation can significantly inhibit osteoinduction. The addition of purified phosphatidylcholine (PC) can restore activity. Furthermore, by adding PC, a natural phospholipid present in bone-forming sites, to active DBM, the biological activity is enhanced above that of a standard DBM preparation..$^{10}$

Laboratory comparison of different DBM products is limited. Using a nude rat (without thymus and without hair) model, Lee et $\mathrm{al}^{11}$ and Wang et $\mathrm{al}^{21}$ compared a number of different commercially available DBM products. Among the products tested, Grafton ${ }^{\circledR}$ DBM putty (Osteotech, Inc, Eatontown, NJ) and Osteofil ${ }^{\circledR}$ allograft bone paste (Regeneration Technologies, Inc, Alachua, Fl) appeared to outperform Dynagraft $^{\circledR}$ putty (Keystone Dental, Burlington, MA). ${ }^{6}$

The use of materials containing different types of growth factors (eg, BMP) is common. In several studies, histology reveals that new bone is generated by osteoconductivity of DBM. ${ }^{8}$ In vitro studies have shown that femtomolar concentrations of BMP initiate chemotaxis of several cell types. Chemotaxis of monocytes occurs by such concentrations of BMP-3 and BMP-4, and BMP-2 was also found to be chemotactic for mature osteoblasts. BMP doses in the nanogram range have shown mitogenic and osteogenic effects in cell culture experiments. However, macroscopic quantities of bone in vivo are induced only by milligram quantities of purified BMP. Finally, BMPs were very effective in animal studies, although the results were very different in human studies. ${ }^{8}$ In 1988, Wozney et al showed that growth factors (eg, BMP and transforming growth factor [TGF]) played the most important role in bone formation. ${ }^{22}$ Therefore, BMPs, especially type 2 and type 4 , were added to many biomaterials. ${ }^{23}$

Bioimplants, containing BMPs such as DBM, are clinically used to repair bone defects because of their ability to stimulate bone regeneration. Because of handling issues, DBM granules 
are often combined with an inert carrier, which reduces DBM content to $40 \%$ or less by volume. Recently, Accell ${ }^{\circledR}$ DBM100 (IsoTis OrthoBiologics, Irvine, CA) has been developed, which uses processed DBM as the carrier, resulting in a DBM content of $100 \%$. In 2007, the purpose of Mhawi et al's investigation was to evaluate the use of Accell for bone defect healing. ${ }^{24}$ In conclusion, these results suggest that Accell DBM100 will be clinically useful in repairing craniofacial bone defects. ${ }^{25}$

Lee et al demonstrated that genetically engineered muscle-derived cells can produce BMP-2 and can substantially enhance the healing of a critical-sized bone defect. ${ }^{11}$

Bone regeneration was dependent on the proteolytic sensitivity of the matrices and their architecture. The cell-mediated proteolytic invasiveness of the gels and entrapment of recombinant human Bone Morphogenetic Protein (rhBMP-2) resulted in efficient and highly localized bone regeneration. ${ }^{26}$

A study was designed to evaluate xenogenic bovine DBM and new xenograft (bovine fetal growth plate) effects on the bone healing process. In conclusion, the results of the study indicate satisfactory healing occurred in rabbit radius defect filled with xenogenic bovine DBM and xenogenic bovine fetal growth plate. Complications were not identified, and healing was faster in two grafting groups. The study showed that the power of two grafted materials is equal, and they can produce new bone in the grafted area with the same mechanical strength. ${ }^{27}$

In 2008, Katz et al performed a study to determine the correlation of specific growth factors and in vitro mitotic stimulation with in vivo ectopic bone formation capacity with a large number of DBM samples. ${ }^{28}$ The results of the study amplify the notion that a multitude of factors and their relative interplay, rather than a single factor, are likely to determine the potency of any particular lot of DBM. These results also highlight the importance of an appropriate assay to qualify unknown DBM preparations with regard to their biological activity.

In 2009, Lee et al evaluated the cellular response for the osteoinductive effect of DBM graft materials by immunohistochemistry. ${ }^{29}$ The study demonstrated that for sinus floor elevation, autogenic bone graft or osteogenic stem cell replacement is necessary, whereas DBM allograft alone is insufficient. It was already known that DBM has an osteoinductive effect stimulating and inducing the osteogenic cells, but the lack of osteogenic cells in sinus mucosa seems to be the direct cause of the implant failure to form the interosseous bony fusion between the implant and DBM allogenic materials at the clinical time of this study.

In 2009 , the results of the study by Kaya et al indicated that either putty or particulate DBM demonstrated similar enhancements in soft and hard tissue parameters. ${ }^{30}$ Applying putty or particulate-form DBM results in slight bone formation when compared with open flap debridement in horizontal bone defects at 1-year postoperative examination, according to bone probing depth measurements.

An increasing number of DBM-based products are commercially available for spinal fusion procedures, but osteoinductive variability has been found not only across different products but also among production lots from the same DBM formulation. The purpose of the study in 2010 by Bae et al was to assess the lot-to-lot variability across a single DBM-based product in terms of both extracted BMP concentrations (in vitro) and fusion performance in rats (in vivo). ${ }^{31}$ They concluded that assays for DBM-extracted BMP-2 and BMP-7 levels may be feasible and sufficient for predicting spinal fusion performance of individual production lots from the same DBM-based product. To improve the consistency and efficacy of DBM-mediated fusions in clinical settings, it may be valuable to perform simple terminal screenings for BMP-2 and BMP-7 on DBM-based product lots prior to their commercial distribution. This method was applied in this study's product, so the results achieved were more reliable.

ITB-DBM has a high amount of different types of BMP and collagens and was analyzed by the Japanese company Nippi Inc (Tokyo, Japan). The results showed the positive effect of ITB-DBM on bone regeneration. The amount of regeneration in the middle of the defect was related to their growth factors and collagens.

Nonunions are severe complications in orthopedic trauma care and occur in $10 \%$ of all fractures. ${ }^{32}$ Also, bone defects in maxillofacial procedures and need for bone grafts are another problem today. There is morbidity associated with the bone-graft donor site, and some patients offer limited quantity or quality of autologous-bone graft material. The current study led to an advanced outcome in the treatment of nonunions and simultaneously to a decreased quantity of adverse effects with DBM. The authors conclude that DBM should be offered as an alternative to autologous bone grafting for the treatment of ununited fractures, including open reduction and internal fixation.

There are some possible disadvantages associated with the use of allografts. As mentioned before, allografts might be the potential immunogenicity compared with autologous grafting, which was shown not only in animal models but probably also in clinical applications. Fortunately, in this study, allograft-related immunological adverse reactions have not been observed, but this potential problem should be considered using DBM, so the authors suggest human trials to understand the in vivo effect of ITB-DBM. 


\section{Acknowledgments}

Funding of this study was provided by the Iranian Tissue Bank Research and Preparation Center, Imam Khomeini Hospital Complex, and Tehran University of Medical Sciences. The authors thank everybody who helped with this study and with the publication of this article. Also, we are grateful to Ms Nassim Khatibi for English language editing of this article.

\section{Disclosure}

The authors report no conflicts of interest in this work.

\section{References}

1. Kraus KH, Kirker-Head C. Mesenchymal stem cells and bone regeneration. Vet Surg. 2006;35:232-242.

2. Vaccaro RA. The role of osteoconductive scaffold in synthetic bone graft. Orthopedics. 2002;25(Suppl 5):80-571.

3. Silber JS, Anderson DG, Daffner SD, et al. Donor site morbidity after anterior iliac crest bone harvest for single-level anterior cervical discectomy and fusion. Spine (Phila Pa 1976). 2003;28(2):134-139.

4. Joshi A, Kostakis GC. An investigation of post-operative morbidity following iliac crest graft harvesting. Br Dent J. 2004;196:167-171.

5. Moore WR, Graves SE, Bain GI. Synthetic bone graft substitute. ANZ J Surg. 2001;71:354-361.

6. Grauer JN, Beiner JM, Kwon BK, et al. Bone graft alternatives for spinal fusion. Biodrugs. 2003;17(6):391-394.

7. Becker W, Becker BE, Caffesse R. A comparison of demineralized freeze-dried bone and autologous bone to induce bone formation in human extraction sockets. J Periodontol. 1994;65:1128-1133.

8. Groeneveld EHJ, Burger EH. Bone morphogenetic proteins in human bone regeneration. Eur J Endocrinol. 2000;142:9-21.

9. Sandhu HS, Khan SN, Suh DY, et al. Demineralized bone matrix, bone morphogenetic proteins, and animal models of spine fusion: an overview. Eur Spine J. 2001;10:S122-S131.

10. Han B, Tang B, Nimni ME. Combined effects of phosphatidylcholine and demineralized bone matrix on bone induction. Connect Tissue Res. 2003;44:160-166.

11. Lee JY, Musgarave D, Pelinkovic D, et al. Effect of bone morphogenetic protein-2-expressing muscle-derived cells on healing of critical-sized bone defects in mice. J Bone Joint Surg. 2001;83-A(7):1032-1039.

12. Gysin R, Wergedal JE, Sheng MH-C, et al. Ex vivo gene therapy with stromal cells transduced with a retroviral vector containing the BMP4 gene completely heals critical size calvarial defect in rats. Gene Ther. 2002;9:991-999.

13. Rosen DB. Cutting edge: lectin-like transcript-1 is a ligand for the inhibitory human NKR-P1A receptor. J Immunol. 2005;175(12): 7796-7799.

14. Orsini G, Scarano A, Degidi M, Caputi S, Iezzi G, Piattelli A. Histological and ultrastructural evaluation of bone around Bio-Oss particles in sinus augmentation. Oral Dis. 2007;13:586-593.

15. Srivastava AK, Libanati C, Oberrauch W, Leenings J, Greenwald M, Baylink DJ. Analytical and clinical evaluation of the Bio-Rad HPLC kit for measurement of type I collagen cross links. J Bone Miner Metab. 2003;21(3):134-144.

Clinical, Cosmetic and Investigational Dentistry

\section{Publish your work in this journal}

Clinical, Cosmetic and Investigational Dentistry is an international, peer-reviewed, open access, online journal focusing on the latest clinical and experimental research in dentistry with specific emphasis on cosmetic interventions. Innovative developments in dental materials, techniques and devices that improve outcomes and patient satisfac-
16. Lim CS, Kim SG, Lim SC. Effect of synthetic $\beta$-TCP and bovine-derived hydroxyapatite grafting on bone regeneration in rats. Key Eng Mater. 2008:361-363, 1261-1264.

17. Wu YC, Shaw SY, Lin HR, et al. Bone tissue engineering evaluation based on rat calvaria stromal cells cultured on modified PLGA scaffolds. Biomaterials. 2006;27:896-904.

18. Czuryszkiewicz-Cyrana J, Banach J. Autogenous bone and platelet-rich. plasma (PRP) in the treatment of intrabony defects. Adv Med Sci. 2006; 51 (Suppl 1):26-30

19. Suba Z, Hrabák K, Huys L, et al. Histologic and histomorphometric study of bone regeneration induced by beta-tricalcium phosphate (multicenter study). Orv Hetil. 2004;145(27):1431-1437.

20. Jassar S, Srivastava D, Srivastava B. Efficacy of various alloplastic materials in bony defects - a clinico radiological study. Int J Oral Maxillofac Surg. 2007;36(11):1008.

21. Wang EA, Rosen V, D'Alesandro JS, et al. Recombinant human bone morphogenetic protein induces bone formation. Proc Natl Acad Sci USA. 1990;87(6):2220-2224.

22. Wozney JM, Rosen V, Celeste AJ, et al. Novel regulators of bone formation: molecular clones and activities. Science.1988;242(4885): $1528-1534$

23. Issa MJP, Petenusci SO, Sissere S, et al. Biochemical analysis by calcium determination on the newly formed bone in critical bone defects submitted to different treatments. A pilot study. Int J Morphol. 2007;25(3):511-518

24. Mhawi AA, Peel SA, Fok TC, Clokie CM. Bone regeneration in athymic calvarial defects with Accell DBM100. J Craniofac Surg. 2007;18(3): 497-503.

25. Amir A, Fitzgerald SA, Chi-On BSc T, et al. Bone regeneration in athymic calvarial defects with Accell DBM100. J Craniofac Surg. 2007;18(3):497-503.

26. Lutolf MP, Weber FE, Schmoekel HG, et al. Repair of bone defects using synthetic mimetics of collagenous extracellular matrices. Nat Biotechnol. 2003;21:513-518.

27. Bigham AS, Dehghani SN, Shafiei Z, et al. Experimental bone defect healing with xenogenic demineralized bone matrix and bovine fetal growth plate as a new xenograft: radiological, histopathological, and biomechanical evaluation. Cell Tissue Bank. 2009;10(1):33-41.

28. Katz JM, Nataraj C, Jaw R, et al. Demineralized bone matrix as an osteoinductive biomaterial and in vitro predictors of its biological potential. J Biomed Mater Res B Appl Biomater. 2009;89(1):127-134.

29. Lee SS, Jang JH, Kim KS, et al. Failure of bone regeneration after demineralized bone matrix allograft in human maxillary sinus floor elevation. Basic Appl Pathol. 2009;2(4):125-130.

30. Kaya Y, Yalim M, Bahçecitapar M, et al. Comparison of applying particulate demineralized bone matrix (DBM), putty DBM and open flap debridement in periodontal horizontal bone defects. A 12-month longitudinal, multi-center, triple-blind, split-mouth, randomized, controlled clinical study. Part 1 - clinical and radiographic evaluation. J Oral Rehabil. 2009 Jul;36(7):524-534.

31. Bae H, Zhao L, Zhu D, et al. Variability across ten production lots of a single demineralized bone matrix product. J Bone Joint Surg Am. $2010 \mathrm{Feb} ; 92: 427-435$.

32. Pieske O, Wittmann A, Zaspel J, et al. Autologous bone graft versus demineralized bone matrix in internal fixation of ununited long bones. J Trauma Manag Outcomes. 2009 Dec 15;3:11.

tion and preference will be highlighted. The manuscript management system is completely online and includes a very quick and fair peerreview system, which is all easy to use. Visit http://www.dovepress. com/testimonials.php to read real quotes from published authors. 